Pacific Journal of Mathematic 


\title{
ANALYTIC AND HARMONIC OBSTRUCTION ON NONORIENTABLE KLEIN SURFACES
}

\author{
Norman L. AlLing
}

It is well-known that, on a finitely connected, noncompact, Riemann surface, the complex-dimension of the space of all analytic differentials modulo the space of exact analytic differentials is the first Betti number of the underlying surface, and hence its real-dimension twice the first Betti number. Further, it is well-known that the group of units of the algebra of analytic functions on such a surface modulo the subgroup of exponential functions is a free Abelian group whose rank is again the first Betti number of the underlying surface. Thus, in each case, the analytic obstruction on the surface fully dualizes the continuous obstruction.

Interestingly, this is not the case on a finitely connected, noncompact, nonorientable Klein surface; for example, in the case of the first problem, the real-dimension is twice the first Betti number minus one. In the second problem, the group in question is isomorphic to the direct sum of the two element group and the free Abelian group whose rank is the first Betti number, of the underlying space, minus one. These calculations are first made using sheaf theory, in $\S 2$. Integration theory is then applied, $\S 3$, to elucidate the reason that this curious defect occurs. Application is then made, using integration, to a mixed harmonic--analytic obstruction problem in $\S 4$. Finally, the Dirichlet deficiency of the analogue of the standard algebra on compact, nonorientable, Klein surfaceswith boundary-is computed. Again the defect of minus one occurs. Throughout, the reason why this defect occurs in the nonorientable case is of prime concern.

o. Foundations. The analytic foundations of the theory of Klein surfaces can be found in [11], [3], and [4]. Further, Greenleaf's paper [7], a companion to this, provides another reference, as well as proving Cartan's Theorem $B$ in this context.

Let $\mathcal{Y}$ be a noncompact, nonorientable, Klein surface, (without boundary), and let $\mathfrak{X} \stackrel{p}{\rightarrow} \mathfrak{Y}$ be its complex double [4]: i.e., $\mathfrak{X}$ is a Riemann surface and $p$ a two-to-one local homeomorphism of $\mathfrak{X}$ onto $\mathfrak{Y}$. Recall also that $\mathfrak{X}$ has an antianalytic involution $\tau$ such that $p \circ \tau=p$. Let $\mathscr{O}$ be the structure sheaf on $\mathfrak{X}, \mathscr{C}$ the constant sheaf on $\mathfrak{X}$, and $\Omega$ the sheaf of germs of analytic differentials on $\mathfrak{X}$. Given an open set $U$ of $Y$ let $\mathscr{H}(U) \equiv\left\{s \in \Gamma\left(p^{-1}(U), O\right): s=\sigma(s)\right\}$, where $\sigma(s)=\kappa \circ s \circ \tau, \kappa$ denoting complex conjugation; thus $\mathscr{\mathscr { C }}$ is a presheaf on $Y$. Let $\mathscr{K}$ be defined by replacing $\mathscr{O}$ by $\mathscr{C}$, $\mathscr{H}^{*}$ be replacing 
$\mathcal{O}$ by $\mathcal{O}^{*}$, and $\mathscr{G}$ by replacing $\mathcal{O}$ by $i Z$.

Lemma 0.1. $\mathscr{H}, \mathscr{K}, \mathscr{H}^{*}$, and $\mathscr{C}$ are complete presheaves.

Let the associated sheaves be denoted by the same symbols and let these sheaves be called, respectively, the sheaf of germs of holomorphic functions on $\mathfrak{Y}$, twisted constant sheaf on $Y$, the sheaf of germs of nonzero holomorphic functions on $\mathfrak{Y}$, and the twisted $i Z$ sheaf on $Y$. Holomorphic differentials can be defined on $\mathfrak{X}$ in a variety of ways. The method used in [4] was to choose $U \equiv\left(U_{j}, z_{j}\right)_{j \in J} \in \mathfrak{X}$ and to associate with it a family $\omega \equiv\left(\omega_{j}\right)_{j \in J}$ of holomorphic functions, $\omega_{j}$ being defined on $U_{j}$ subject to an appropriate compatability condition. We then passed to equivalence classes, etc. Let $\sigma(\omega) \equiv\left(\sigma\left(\omega_{j}\right)\right)_{j \in J}$. The following can be proved easily.

LEMMA 0.2. $\sigma(w)$ is a holomorphic differential on $\mathfrak{X}$. Further if $\omega=f d g, f$ and $g$ holomorphic on $\mathfrak{X}$, then $\sigma(\omega)=\sigma(f) d \sigma(g)$.

Let $\Delta(U) \equiv\left\{\omega \in \Gamma\left(p^{-1}(U), \Omega\right): \omega=\sigma(\omega)\right\}$; then it is easily seen that $\Delta$ is a complete presheaf. Let $\Delta$ be the sheaf on $Y$ associated with this presheaf. Then it is easily seen that $\Delta$ is a locally free sheaf of $\mathscr{X}$-modules of rank one on $Y$, and that following sequence of sheaves and sheaf maps is exact:

$$
0 \longrightarrow \mathscr{C} \longrightarrow \mathscr{C} \stackrel{d}{\longrightarrow} \Delta \longrightarrow 0 \text {. }
$$

The de Rham problem, in this context, is the following: find $\operatorname{dim}_{R} \Delta(\mathfrak{Y}) / d \mathscr{H}(\mathfrak{Y})$. Applying the cross section functor to $(0 . \mathrm{a})$, we arrive at the following long exact sequence:

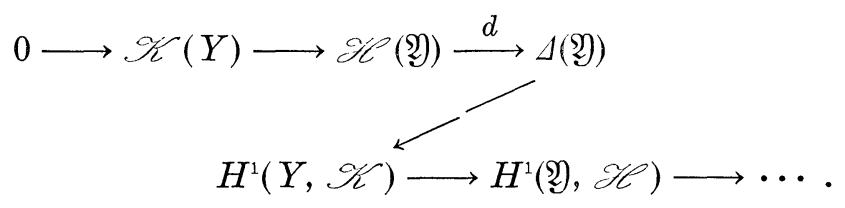

Given an open set $W$ in $\mathfrak{X}$ and $s \in \Gamma(W, \mathcal{O})$, let $\exp s \equiv e^{2 \pi s}$. The following sequence of sheaves and sheaf maps on $\mathfrak{X}$ is exact.

$$
0 \longrightarrow i Z \longrightarrow \mathcal{\longrightarrow} \stackrel{\exp }{\longrightarrow} \mathcal{O}^{*} \longrightarrow 1 \text {. }
$$

It is easily seen that this sequence induces the following exact sequence of sheaves and sheaf maps on $\mathfrak{Y}$.

$$
0 \longrightarrow \mathscr{G} \longrightarrow \mathscr{H} \stackrel{\exp }{\longrightarrow} \mathscr{H}^{*} \longrightarrow 1 \text {. }
$$

Applying the cross section functor to $(0 . d)$ results in the following 
long exact sequence:

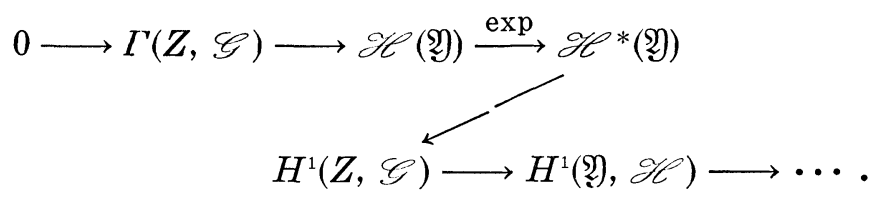

This sequence will be used to analyze $\mathscr{C}^{*}(\mathfrak{Y}) / \exp \mathscr{\mathscr { C }}(\mathfrak{V})$, the group of units modulo the subgroup of exponentials.

1. Introduction. Having the long de Rham cohomology sequence $(0 . b)$ and the long exponential cohomology sequence (0.e), let us analyze them, beginning with the first interesting terms, $\mathscr{K}(Y)$ and $\Gamma(Y, \mathscr{G})$, by noting that these groups depend only on the topology on $Y$.

We assumed at the outset that $Y$ is connected. If $Y$ is orientable, $\mathscr{K}$ is just the constant sheaf $\mathscr{C}$, so that $\mathscr{K}(Y) \simeq{ }_{R} C$ and $\Gamma(Y, \mathscr{G}) \simeq i Z$. If $Y$ is nonorientable, $X$ is connected and, since $\mathscr{K}(Y)=\{s \in \Gamma(X, \mathscr{C}): s=\sigma(s)\}, \mathscr{K}(Y)=R$ and $\Gamma(Y, \mathscr{C})=0$. Thus we have the following.

Proposition 1.1. If $Y$ is orientable $\mathscr{C}(Y) \simeq{ }_{R} C$ and $\Gamma(Y, \mathscr{G}) \simeq$ iZ. However, if $Y$ is nonorientable $\mathscr{K}(Y)=R$ and $\Gamma(Y, \mathscr{G})=0$; thus exp in (0.e) is injective in this case.

The following essential theorem is an easy consequence of a very special case of Cartan's Theorem B.

Theorem. $\left(\right.$ Cartan) 1.2. $H^{1}(\mathfrak{Y}, \mathscr{C})=0$.

Note. In the companion paper by Greenleaf [7] he gives a much more general result. The proof is included here only to make this paper more self-contained.

Proof. In case $Y$ is orientable choose an analytic structure $\mathfrak{Y}_{1} \subset \mathfrak{V}$. Since every Riemann surface is a Stein manifold $H^{1}\left(\mathfrak{Y}_{1}, \mathscr{C}\right)=0$. Assume now that $Y$ is nonorientable. By swelling out a triangulation of $Y$ a bit we can choose $\boldsymbol{U} \equiv\left(U_{j}, z_{j}\right)_{j \in J} \in \mathfrak{V}$ that is locally finite such that each $U_{j}$ and each $U_{j} \cap U_{k}$ is simply connected and so that each $p^{-1}\left(U_{j}\right)$ has two components. Through an abuse of notation, let $\boldsymbol{U}$ also denote $\left(U_{j}\right)_{j \in J}$ and let $V \equiv\left(p^{-1}\left(U_{j}\right)\right)_{j \in J}$, and note that $V$ is a locally finite covering of $X$ which is a Leray relative to $\mathcal{O}$. Every 1 -cocycle (resp. bounding 1-cocycle) on $U$ with coefficients in $\mathscr{C}$ is a 1-cocycle (resp. bounding 1-cocycle) on $V$ with coefficients in $\mathcal{O}$. Thus these inclusion maps induce an $R$-linear map $I_{U}$ of $H^{1}(U, \mathscr{C})$ into $H^{1}\left(\boldsymbol{V}, \mathcal{O}^{\circ}\right)$. Since $\sigma$ is an $R$-linear involution of $\Gamma\left(p^{-1}\left(U_{j}\right), \mathcal{C}\right)$, it 
induces an $R$-linear involution $\sigma$ of $H^{1}(\boldsymbol{V}, \mathcal{O})$ which has $I_{\boldsymbol{U}}\left(H^{1}(\boldsymbol{U}, \mathscr{C})\right)$ as its set of fixed points. Let $T_{U} \equiv(1+\sigma) / 2$, and note that $T_{U} I_{U}$ is the identity map of $H^{1}(\boldsymbol{U}, \mathscr{C})$; thus $I_{U}$ is injective. By Cartan's Theorem B (see e.g., [8] for details), $H^{1}(\boldsymbol{V}, \mathcal{O})=0$, proving the theorem.

Note: To define the normalized trace map $T_{U}$ we divided by 2 . Thus it is not a-priori true that $I_{\boldsymbol{U}}$ maps $H^{1}(\boldsymbol{U}, \mathscr{G})$ injectively into $H^{1}(\boldsymbol{V}, i Z)$. Were $I_{U}$ injective in this case $H^{1}(Y, \mathscr{G})$ would be torsion free.

COROLlARY 1.3. The following sequences are exact:

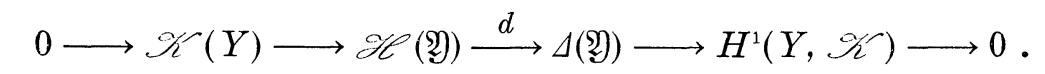
$0 \longrightarrow \Gamma(Y, \mathscr{C}) \longrightarrow \mathscr{C}(\mathscr{O})$
(Y) $\stackrel{\exp }{\longrightarrow} \mathscr{C}^{*}(\mathfrak{Y}) \longrightarrow H^{1}(Y, \mathscr{C}) \longrightarrow 0$.

COROLlary (Mittag-Le.fler, Florack) 1.4. The Mittag-Leffler theorem holds on $\mathfrak{Y}$.

Proof (i). If $Y$ is orientable use Florack's argument, in which she invokes Theorem B [6]. If $Y$ is nonorientable, use that fact that $H^{1}(\mathfrak{Y}, \mathscr{X})=0$, as Florack did.

Proof (ii). In the nonorientable case, choose one singularity in $X$ over each singularity in $Y$, choose appropriate principal parts at these singularities in $X$, use Florack's result to obtain a global meromorphic function on $X$ having these singularities; on taking its trace one is finished.

COROLlaRY (Weierstrass, Florack) 1.5. The Weierstrass "product" theorem holds on $\mathfrak{Y}$.

Of course, if $Y$ is orientable, this is nothing other than Florack's Theorem [6]. Assume that $\mathfrak{Y}$ is nonorientable. To utilize her argument here we need to know that $H^{1}\left(Y, \mathscr{C}^{*}\right)=0$. If we knew that $H^{2}(Y, \mathscr{G})=0$ we could use $(0 . \mathrm{e})$ to achieve this. This produces no obstacle, however, since the analogue of Proof (ii) above easily proves Corollary 1.5 .

2. Čech cohomology on finitely connected klein surfaces and applications. In $\S 1$ the problem of computing $\operatorname{dim}_{R} \Delta(\mathfrak{Y}) / d(\mathscr{H}(\mathfrak{Y}))$ and $\mathscr{C}^{*}(\mathfrak{Y}) / \exp \mathscr{H}(\mathfrak{Y})$ was reduced to computing $\operatorname{dim}_{R} H^{1}(Y, \mathscr{K})$ and $H^{1}(Y, \mathscr{C})$ in (1.3). Let us restrict our attention to a Klein surface $\mathfrak{Y}$ 
which is the interior of a compact Klein surface $\overline{\mathfrak{Y}}$ that has a nonempty boundary $\partial \bar{Y}$. Since $H^{1}(Y, \mathscr{K})$ and $H^{1}(Y, \mathscr{C})$ do not depend on the dianalytic structure $\mathfrak{Y}$, but depend only on the topology of $Y$-as the notation suggests - we can compute these group on spaces homeomorphic to $Y$; thus the initial results of this section deal only with topology, although analysis may creep into some of the proofs.

Let $k$ be the number of components of $\partial \bar{Y}$; thus $k$ is an integer, $k \geqq 1$. It is well-known (see e.g., [9] as a general reference here), that $\bar{Y}$ is characterized by knowing the following data: If it is orientable or nonorientable, $k$, and $\chi(\bar{Y})$-the Euler characteristic of $\bar{Y}$. Let $\bar{D} \equiv\{z \in C:|z| \leqq 1\}$. If $\bar{Y}$ is nonorientable, adjoin a halftwisted strip to $\bar{D}$ to form a Möbius strip $\bar{D}_{1}$; if not, let $\bar{D}_{1} \equiv \bar{D}$. (Again see [9].) Now adjoin $k-1$ untwisted strips to $\bar{D}_{1}$ to form $\bar{D}_{2}$. Now adjoin $h$ handles to $\bar{D}_{2}$ in the form of pairs of interlocking untwisted strips, to form $\bar{D}_{3}$ so that $\chi\left(\bar{D}_{3}\right)$ is reduced to either $\chi(\bar{Y})$ or to $\chi(\bar{Y})+1$. In the first case let $\bar{D}_{4} \equiv \bar{D}_{3}$, and in the second let $\bar{D}_{4}$ be formed from $\bar{D}_{3}$ by adjoining a half-twisted strip; so that $\chi\left(\bar{D}_{4}\right)=\chi(\bar{Y})$ in either case. Thus $\chi\left(\bar{D}_{4}\right)=1-(k-1)-2 h-m$, where $m$ is the number of half-twisted strips adjoined. $m=0,1$, or 2 , the surface being orientable if and only if $m=0$. That each case arises may be seen by letting $\bar{Y}=\bar{D}$, letting $\bar{Y}$ be a Möbius strip, and letting $\bar{Y}$ be a disc with two half twisted adjoined. Clearly $k$ and $h$ can assume any integer $k \geqq 1$ and $h \geqq 0$. To summarize:

$$
\chi(Y)=1-(k-1)-2 h-m,
$$

the integers $k, h$ and $m$ being a complete set of invariants for $Y$. Let $b(Y)$ be the first Betti number of $Y$. Then

$$
b(Y)=k-1+2 h+m .
$$

Proposition 2.1. Assume that $Y$ is orientable; then $\operatorname{dim}_{R} H^{1}(Y$, $\mathscr{K})=2 b(Y)$ and $H^{1}(Y, \mathscr{C})$ is a free Abelian group of rank $b(Y)$.

Proof. In this case $\mathscr{K}$ is isomorphic to the constant sheaf $C$ and $\mathcal{G}$ to the constant sheaf $i Z$, proving the proposition.

Assume that $Y$ is nonorientable; then $X$, its complex double, is connected. If $m=1, b(X)=2(k-1)+4 h+1$, and if $m=2, b(X)=$ $2(k-1)+4 h+3$. Hence

$$
b(X)=2(k-1)+4 h+m(m+1) / 2, \quad m=1,2 .
$$

Lemma 2.2. Assume that $Y$ is nonorisntable. $\operatorname{dim}_{R} H^{1}(Y, \mathscr{K})=$ $\frac{1}{2} \operatorname{dim}_{R} H^{1}(X, \mathscr{C})=\operatorname{dim}_{R} H^{1}(X, \mathscr{R})$. 
Proof. The injection $I$ of $\mathscr{K}$ into $\mathscr{C}$ and the trace $T(\equiv(1+\sigma) / 2)$ of $\mathscr{C}$ onto $\mathscr{\mathscr { C }}$ induce the following maps:

$$
H^{1}(Y, \mathscr{K}) \underset{T}{\stackrel{I}{\rightleftarrows}} H^{1}(Y, \mathscr{C}) \text {. }
$$

Since $T I$ is the identity map, $I$ is injective. Since the elements of $R, \mathscr{C}(\mathfrak{Y})$, and $\Delta(\mathfrak{Y})$ together with $i$ times these elements-respectively-generate $C, \mathscr{O}(\mathfrak{X})$, and $\Omega(\mathfrak{X})$-respectively; $\operatorname{dim}_{R} H^{1}(X, \mathscr{C})=$ $2 \operatorname{dim}_{R} H^{1}(Y, \mathscr{K})$, proving the lemma.

THEOREM 2.3. $\operatorname{dim}_{R} H^{1}(Y, \mathscr{K})$ is $2 b(Y)$ if $Y$ is ori气ntable, and $2 b(Y)-1$ if $Y$ is nonorientable. In terms of $k, h$, and $m, \operatorname{dim}_{R} H^{1}(Y$, $\mathscr{K})=2(k-1)+4 h+m(m+1) / 2$.

At this stage, it is not immediately clear why in the nonorientable case, the analytic obstruction, as evidenced by the solution of the de $\mathrm{Rhm}$ problem in this case, is one less than the topological obstruction. One of the main objectives of this paper is not only to compute the analytic obstruction on nonorientable $Y$, but also to account for the defect form what is expected from the topological obstruction.

Let us turn our attention to $H^{1}(Y, \mathscr{G})$.

ExAMPle 2.1. Let $Y$ be a Möbius strip. Let $Y$ be written as follows:

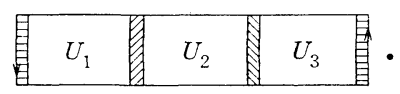

Let $z_{j}$ be a coordinate function on $U_{j}$, so that all transition functions are orientation preserving expect $z_{1} z_{3}^{-1}$ and $z_{3} z_{1}^{-1}$. Let $\boldsymbol{U} \equiv\left(U_{1}, U_{2}, U_{3}\right)$. Let 0 -cocycles on $\boldsymbol{U}$ with coefficients in $\mathscr{G}$ be written as $\left(f_{1}, f_{2}, f_{3}\right)$. The coboundary of $\left(f_{1}, f_{2}, f_{3}\right), \partial\left(f_{1}, f_{2}, f_{3}\right)$ is the 1-cocycle $\left(f_{2}-f_{1}\right.$, $\left.f_{3}-f_{2}, f_{1}-f_{3}\right)$. Let $f_{1}-f_{3}$ be written in terms of $z_{1}$. Clearly $H^{1}(\boldsymbol{U}, \mathscr{G})$ is cyclic, being generated by the 1-cocycle $(0,0, i)$. Further $\partial(i, i, i)=$ $(0,0,2 i)$; thus $H^{1}(\boldsymbol{U}, \mathscr{C})$, and also $H^{1}(Y, \mathscr{C})$, is isomorphic to $Z_{2}$.

THEOREM 2.4. If $Y$ is orientable, $H^{1}(Y, \mathscr{G}) \simeq Z^{b\left(Y^{*}\right)}$, and if $Y$ is nonorientable $H^{1}(Y, \mathscr{C}) \simeq Z_{2} \oplus Z^{b\left(Y^{Y}\right)-1}$.

Proof. Assume that $Y$ is orientable; then $H^{1}(Y, \mathscr{C}) \simeq H^{1}(Y, Z) \simeq$ $Z^{b(Y)}$. Assume that $Y$ is nonorientable. If $m=1$ we can treat the twisted and untwisted strips adjoined to $\bar{D}_{1}$ to form $\bar{D}_{4}$ independently and arrive at the results above. Assume that $m=2$, and consider the following example. 
EXAMPLE 2.2. Let $\bar{Y}$ be formed from $\bar{D}$ by adjoining two halftwisted strips thus $k=1, h=0$, and $m=2$. Let $U_{1}$ be the interior of $\bar{D}$. Let $U_{2} \cup U_{3}$ form one half-twisted strip of $Y, U_{1} \cap U_{2}, U_{1} \cap U_{3}$, and $U_{2} \cap U_{3}$ being simply connected. Similarly, let $U_{4} \cup U_{5}$ make up the other half-twisted strip on $Y$ subject to the same restraints. Let $z_{2} z_{3}^{-1}, z_{3} z_{2}^{-1}, z_{4} z_{5}^{-1}$, and $z_{5} z_{4}^{-1}$ be the only orientation reversing transition functions, the family $\left(U_{j}, z_{j}\right)_{j \in\{1,2,3,4,5\}} \equiv \boldsymbol{U}$ being an atlas of $Y$. Let 1-cocycles be written $\left(f_{12}, f_{23}, f_{31} ; f_{14}, f_{45}, f_{51}\right)$; thus $\alpha \equiv(0, i, 0 ; 0,0,0)$ and $\beta \equiv(0,0,0 ; 0, i, 0)$ generate $H^{1}(\boldsymbol{U}, \mathscr{C})$. Observing the same conventions as were adopted in Example 2.1, the coboundary of the 0 -cocycle $(i, i, i, i, i)$ is $(0,2 i, 0 ; 0,2 i, 0)$ : i.e., $2\left(a^{\prime}+\beta\right)=0$. Hence $H^{1}(\boldsymbol{U}, \mathscr{C})$ is isomorphic to $Z \times Z /(2,2) Z$ : i.e., $H^{1}(\boldsymbol{U}, \mathscr{G}) \simeq Z_{2} \oplus Z$. We conclude that $H^{1}(Y, \mathscr{C}) \simeq Z_{2} \oplus Z$.

Returning now to the proof of (2.4), note that applying Example 2.2 to the case at point, $H^{1}(Y, \mathscr{G}) \simeq\left(Z_{2} \oplus Z\right) \oplus Z^{b(Y)-2} \simeq Z_{2} \oplus Z^{b(Y)-1}$, since the twisted and untwisted strips may be treated independently; this proves the theorem.

Having analyzed $H^{1}(Y, \mathscr{K})$ and $H^{1}(Y, \mathscr{C})$, let us synthesize the results of $\S 1$ and $\S 2$ to form the main theorem of this section.

THEOREM 2.5. $\operatorname{dim}_{R} \Delta(\mathfrak{Y}) / d \mathscr{Y}(\mathfrak{Y})$ is $2 b(Y)$ or $2 b(Y)-1$ according as $Y$ is orientable or not. $\mathscr{H}^{*}(\mathfrak{Y}) / \exp \mathscr{Y}(\mathfrak{Y})$ is isomorphic to $Z^{b(Y)}$ or to $Z_{2} \oplus Z^{b\left(Y^{Y}\right)-1}$ according as $Y$ is orientable or not.

It may come to the reader as a surprise to see that $\mathscr{H}^{*}(\mathfrak{Y}) /$ $\exp \mathscr{C}(\mathfrak{Y})$ has a nontrivial torsion group in case $Y$ is nonorientable. It is natural to wish to find a generator of this group.

TheOREM 2.6. $Y$ is orientable if and only if $-1 \in \exp \mathscr{X}$ (Y). In case $Y$ is nonorientable the residue of -1 in $\mathscr{H}^{*}(\mathfrak{Y}) / \exp \mathscr{H}$ (Y) generates its torsion group, and is order 2.

Proof. Assume that $Y$ is orientable, then $i \in \mathscr{C}$ (פ) and exp $i / 2=$ -1. Conversely, assume for a moment that there exists $f \in \mathscr{H}$ (Y) such that $\exp f=-1$, and that $Y$ is nonorientable. Recall that $\mathscr{H}(\mathfrak{Y}) \subset \mathscr{H}(\mathfrak{X})$. Since $\exp f=-1$, given $x \in X, f(x)=i / 2+n i$, for some fixed integer $n$. Since $X$ is connected $f=i / 2+n i$. Since $f \in \mathscr{L}(\mathfrak{Y}), f=\sigma(f)$ : i.e., $i / 2+n i=\kappa \circ(i / 2+n i)=-i / 2-n i$ or $n=-1 / 2$; which is absurd, proving that $Y$ nonorientable implies $-1 \notin \exp \mathscr{\mathscr { C }}$ (Y)). The rest of (2.6) follows (2.5), and can also be proved independently with ease.

3. Integration and obstruction on $\mathfrak{Y}$. If $\mathfrak{Y}$ is orientable one way to approach the de Rham problem is by defining a bilinear pairing 
between $\Delta(\mathfrak{Y}) / d \mathscr{H}(\mathfrak{Y})$ and $H_{1}(Y, C)$, the first singular homology group on $Y$ with complex coefficients, with the aid of integration.

Until we have achieved the main theorem of this section, (3.6), assume that $Y$ is nonorientable. In this case the integration theory of analytic differentials $\omega$ along arcs and curves on $\mathfrak{Y}$ exists [4]; however, it requires care to apply it. For example, if $\Gamma$ is an oriented, Jordan curve (see, e.g., [10]) in $\mathfrak{Y}$ which can be covered by a finite number of dianalytic charts $\boldsymbol{U} \equiv\left(U_{j}, z_{j}\right)_{j \in J}$, all of whose transition functions are analytic, then $\int_{\Gamma} \omega$ is a well defind complex modulo the choice of $\boldsymbol{U}$. Such a curve will be called an even curve in $\mathfrak{Y}$. Note that $\int_{\Gamma} \boldsymbol{\omega}$ is dependent on the choice of $\boldsymbol{U}$ in that if $\bar{U} \equiv\left(U_{j}, \bar{z}_{j}\right)_{j \in J}$ is chosen instead, the resulting integral is the conjugate of the former. These are also the only possibilities. An oriented Jordan curve $\Gamma$ in $Y$ which has no such covering will be called an odd curve in $\mathfrak{Y}$. Let $\Gamma$ be such a curve and let $y_{0} \in \Gamma$. Let $y \in \Gamma-\left\{y_{0}\right\}$ and note that $\Gamma_{y_{0}}^{y}$, the arc from $y_{0}$ to $y$ in $\Gamma$, can be covered by such an oriented coordinate system; thus $\int_{\Gamma_{y_{0}}^{y}} \omega$ is well defined up to complex conjugation. Letting $y$ approach $y_{0}$ by passing around $r$ and taking the limit defines a complex number $\int_{\Gamma, y_{0}} \omega$ uniquely, up to conjugation and the choice of $y_{0}$. Fortunately $\operatorname{Re} \int_{\Gamma, y_{0}} \omega$ is uniquely determined by $\Gamma$ and $\omega$ and will be denoted by $\operatorname{Re} \int_{\Gamma, y_{0}} \omega$. (For further details see [4]).

There exists a basis $\Gamma_{1}, \cdots, \Gamma_{b(Y)}$ of the first singular homology group on $Y$ such that $\Gamma_{1}, \cdots, \Gamma_{b\left(Y^{\prime}\right)-1}$ are even curves and $\Gamma_{b\left(Y^{\prime}\right)}$ is an odd curve. These curves may be chosen as follows: If $m=1$ let $\Gamma_{1}, \cdots, \Gamma_{b(Y)-1}$ be paths that go around the untwisted strips of $Y$ and let $\Gamma_{b(Y)}$ go around the twisted strip of $Y$. In case $m=2$ let $\Gamma_{1}, \cdots, \Gamma_{b(Y)-2}$ go around the untwisted strips of $Y$, let $\Gamma_{b(Y)-1}$ go around both twisted strips, once each, and let $\Gamma_{b(Y)}$ go around one of the twisted strips. Let the following be defined.

$$
\begin{aligned}
\varphi_{1}(\omega) & \equiv \operatorname{Re} \int_{\Gamma_{1}} \omega / 2 \pi \\
\varphi_{2}(\omega) & \equiv \operatorname{Im} \int_{\Gamma_{1}} \omega / 2 \pi \\
& \vdots \\
\varphi_{2 b(Y)-3}(\omega) & \equiv \operatorname{Re} \int_{\Gamma_{b(Y)-1}} \omega / 2 \pi \\
\varphi_{2 b(Y)-2}(\omega) & \equiv \operatorname{Im} \int_{\Gamma_{b(Y)-1}} \omega / 2 \pi \\
\varphi_{2 b(Y)-1}(\omega) & \equiv \operatorname{Re} \int_{\Gamma_{b(Y)}} \omega / \pi .
\end{aligned}
$$


Note that $\varphi_{1}, \varphi_{3}, \cdots, \varphi_{2 b(Y)-1}$ are uniquely determined by the given data (namely $\Gamma_{1}, \cdots, \Gamma_{b(Y)}$ ), whereas $\varphi_{2}, \varphi_{4}, \cdots, \varphi_{2 b(Y)-2}$ are determined only up to sign, the ambiguity of this choice being independent one from the other.

An alternative to integrating differentials on $\mathfrak{Y}$, using the theory developed in [4], is to integrate $\omega \in \Delta(\mathfrak{Y )})$ on $\mathfrak{X}$. For $1 \leqq j \leqq b(Y)-1$, $\Gamma_{j}$ lifts to two oriented Jordan curves $\Gamma_{j}^{\prime}$ and $\Gamma_{j}^{\prime \prime}$, which are mappedone onto the other, preserving orientation-by $\tau . p^{-1}\left(\Gamma_{b(Y)}\right)$ is also an oriented Jordan curve in $X$.

Lemma 3.1. Let $\Gamma$ be an oriented Jordan curve (arc) in $\mathfrak{X}$ and let $\omega \in \Delta(\mathfrak{Y )})$; then $\int_{\Gamma} \omega=\kappa \circ \int_{\tau(\Gamma)} \omega$.

Proof. This reduces to a local question for any subarc of $I$, and its image under $\tau$. Let $x_{0} \in \Gamma$. We may choose $z \in \mathscr{H}(\mathfrak{Y})$ having a simple zero at $x_{0}$, and necessarily also a simple zero at $\tau\left(x_{0}\right)$, by-for example-the Weierstrass "product" theorem for $\mathfrak{Y}(1.5)$. $\omega$ may be written in the form $f d z, f$ being in $\mathscr{Y}$ (פ). $z$ is a local homeomorphism at $x_{0}$ (and also at $\tau\left(x_{0}\right)$ ). Let $U$ be an open set of $x_{0}$ in $X$ on which $z$ is injective. Let $x_{1}$ be in the component of $\Gamma \cap U$ which contains $x_{0}$. (Necessarily $\tau\left(x_{1}\right)$ is in the component of $\tau(\Gamma) \cap \tau(U)$ which contains $\tau\left(x_{0}\right)$.) Since $f$ and $z$ are symmetric $\left(f\left(x_{1}\right)-f\left(x_{0}\right)\right)\left(z\left(x_{1}\right)\right)-\left(z\left(x_{0}\right)\right)=$ $\left(\sigma(f)\left(x_{1}\right)-\sigma(f)\left(x_{0}\right)\right)\left(\sigma(z)\left(x_{1}\right)-\sigma(z)\left(x_{0}\right)\right)=\left(\kappa \circ f\left(\tau\left(x_{1}\right)\right)-\kappa \circ f\left(\tau\left(x_{0}\right)\right)\right)\left(\kappa \circ z\left(\tau\left(x_{1}\right)\right)-\right.$ $\left.\kappa \circ z\left(\tau\left(x_{0}\right)\right)\right)=\kappa \circ\left[\left(f\left(\tau\left(x_{1}\right)\right)-f\left(\tau\left(x_{0}\right)\right)\right)\left(z\left(\tau\left(x_{1}\right)\right)-z\left(\tau\left(x_{0}\right)\right)\right)\right]$. Since we are dealing with a Riemann-Stieltjes integral, this proves the lemma.

COROLlary 3.2. Let $\omega \in \Delta(\mathfrak{Y})$; then $\int_{\Gamma_{j}^{\prime}} \omega=\kappa \int_{\Gamma_{j}^{\prime}} \omega$, for $1 \leqq j \leqq$ $b(Y)-1, \operatorname{Re} \int_{p^{-1}\left(\Gamma_{b}\langle Y)\right.} \omega=2 \operatorname{Re} \int_{\Gamma_{b(Y)}} \omega$, and $\operatorname{Im} \int_{p^{-1}\left(\Gamma_{b}(Y)\right)} \omega=0$.

We will see that the last fact cited above is a revealing reflection of the reason that the defect occurs in the nonorientable case. The following is easily proved.

Lemma 3.3. $\Gamma_{1}^{\prime}, \Gamma_{1}^{\prime \prime}, \Gamma_{2}^{\prime}, \Gamma_{2}^{\prime \prime}, \ldots, \Gamma_{b(Y)-1}^{\prime}, \Gamma_{b(Y)-1}^{\prime \prime}$ and $p^{-1}\left(\Gamma_{b(Y)}\right)$ constitute a basis for the first singular homology group on $X$.

Let the following be defined

$$
\begin{aligned}
& \theta_{1}(\omega) \equiv \operatorname{Re} \int_{\Gamma_{1}^{\prime}} \omega / 2 \pi \\
& \theta_{2}(\omega) \equiv \operatorname{Im} \int_{\Gamma_{1}^{\prime}} \omega / 2 \pi
\end{aligned}
$$




$$
\begin{aligned}
& \theta_{2 b(Y)-3}(\omega) \equiv \operatorname{Re} \int_{\Gamma_{b(Y)-1}^{\prime}} \omega / 2 \pi \\
& \theta_{2 b(Y)-2}(\omega) \equiv \operatorname{Im} \int_{\Gamma_{b(Y)-1}^{\prime}} \omega / 2 \pi \\
& \theta_{2 b(Y)-1}(\omega) \equiv \operatorname{Re} \int_{p^{-1}\left(\Gamma_{b(Y)}\right)} \omega / 2 \pi .
\end{aligned}
$$

With the aid of integration theory developed in [4], we have the following.

PROPOSITION 3.4. $\theta_{1}=\varphi_{1}, \theta_{2}= \pm \varphi_{2}, \cdots, \theta_{2 b(Y)-2}= \pm \varphi_{2 b(Y)-2}, \theta_{2 b(Y)-1}=$ $\varphi_{2 b(Y)-1}$, the signs for even subscripts being independent of one another.

Lemma 3.5. Let $\omega \in \Delta(\mathfrak{Y})$ such that

$$
\varphi_{1}(\omega)=\varphi_{2}(\omega)=\cdots=\varphi_{2 b(Y)-1}(\omega)=0,
$$

then $\omega \in d \mathscr{L}(\mathfrak{Y})$; and conversely.

Proof. $\omega$ is in $\Omega(\mathfrak{X})$. By (3.2), (3.3), and (3.4) all of its periods are zero. Thus there exists $f \in \mathscr{O}(\mathfrak{X})$ such that $d f=\omega$. Let $g \equiv$ $(f+\sigma(f)) / 2 \in \mathscr{H}(\mathfrak{Y})$, and note that $d g=(\omega+\sigma(\omega)) / 2=2 \omega / 2=\omega$, proving the lemma.

Using (3.5) we see that $\varphi_{1}, \varphi_{2}, \cdots, \varphi_{2 b(Y)-1}$ induce $R$-linear functionals $\widetilde{\varphi}_{1}, \widetilde{\varphi}_{2}, \cdots, \widetilde{\varphi}_{2 b(Y)-1}$ on $\Delta(\mathfrak{Y}) / d \mathscr{H}(\mathfrak{Y})$. Since, as we saw in (2.3), $\operatorname{dim}_{R} \Delta(\mathfrak{Y}) / d \mathscr{C}(\mathfrak{Y})=2 b(Y)-1$, we have arrived at the main theorem of this section.

THEOREM 3.6. $\left\{\widetilde{\varphi}_{1}, \cdots, \widetilde{\varphi}_{2 b(Y)-1}\right\}$ is a basis of $\operatorname{Hom}_{R}(\Delta(\mathfrak{Y )})) / d \mathscr{H}(\mathfrak{Y})$, $R)$.

Let us now turn our attention to $\mathscr{C}^{*}(\mathfrak{Y}) / \exp \mathscr{C}(\mathfrak{Y})$. Assume, at the outset, that $Y$ may be orientable.

Given $g \in \mathscr{C}^{*}(\mathfrak{Y})$, let $\lambda(g) \equiv d g / g$; then $\lambda$ is a homomorphism of the multiplicative group of $\mathscr{C}^{*}(\mathfrak{Y})$ into the additive group $\Delta(\mathfrak{Y})$. The following sequence of Abelian groups and group homomorphisms is exact, $\mathscr{K} *(Y)$ being the multiplicative group of units of the field of constants of $\mathscr{H}(\mathfrak{Y})$; namely $R^{*}$ if $Y$ is nonorientable and $C^{*}$ if $Y$ is orientable:

$$
1 \longrightarrow \mathscr{K}^{*}(Y) \longrightarrow \mathscr{C}^{*}(\mathfrak{Y}) \stackrel{\lambda}{\longrightarrow} \Delta(\mathfrak{Y}) \text {. }
$$

Given $f \in \mathscr{X}(\mathfrak{Y}), \lambda(\exp f)=2 \pi d f$; thus $\lambda(\exp \mathscr{\mathscr { C }}(\mathfrak{Y})) \subset d \mathscr{X}(\mathfrak{Y})$. This shows that $\lambda$ induces a homomorphism $\tilde{\lambda}$ making the following sequence exact. 


$$
1 \longrightarrow K \longrightarrow \mathscr{K}^{*}(\mathfrak{Y}) / \exp \mathscr{\mathscr { C }}(\mathfrak{Y}) \stackrel{\tilde{\lambda}}{\longrightarrow} \Delta(\mathfrak{Y}) / d \mathscr{L}(\mathfrak{Y})
$$

Lemma 3.7. Let $g \in \mathscr{Z}^{*}(\mathfrak{Y})$ such that $d g / g(\equiv \lambda(g))=d f$, for some $f \in \mathscr{H}(\mathfrak{Y})$. Then there exists $c \in \mathscr{K}^{*}(Y)$ such that $g=c \exp f$.

Proof. Let $k \equiv(\exp f) / 2 \pi$, and note that $\lambda(k)=2 \pi(\exp f) d f / 2 \pi \exp f=$ $d f$. Hence $\lambda(g)=\lambda(k)$. Note that $(1 / g k)(d(g / k))=(1 / g k)(k d g-g d k) / k^{2}=$ $\left(1 / k^{2}\right)(d g / g-d k / k)=0$. We conclude that $g / k \equiv c_{0} \in \mathscr{K}^{*}(Y)$, so $g=$ $c_{0} k=\left(c_{0} / 2 \pi\right) \exp f \equiv c \exp f$, proving the lemma.

As an immediate consequence of this lemma we have the following.

Proposition 3.8. If $Y$ is orientable, $K\left(\equiv \tilde{\lambda}^{-1}(0)\right)$ is $\{1\}$. If $Y$ is nonorientable $K=\{ \pm 1\}$.

Proof. $\mathscr{K}^{*}(Y) / \exp \mathscr{K}(Y)$ is isomorphic to $C^{*} / \exp C$ or $R^{*} / \exp R$ according as $Y$ is orientable or not: i.e., it is $C^{*} / C^{*}$ or $R^{*} /\{r \in R: r>0\}$. Using this and (3.7), the proposition is proved.

Note. (3.8) may be considered an arithmetic characterization of orientability or the lack thereof on $Y$.

Assume, until further notice, that $Y$ is nonorientable.

Lemma 3.9. For all $g \in \mathscr{P}^{*}(\mathfrak{Y})$, and all $j, 1 \leqq j<b(Y)$, $1 / 2 \pi \int_{\Gamma_{j}} d g / g \in i Z . \quad$ If $g= \pm \exp f$, for $f \in \mathscr{C}(\mathfrak{Y})$, then the integral above is zero. Finally, $\operatorname{Re}\left(1 / \pi \int_{\Gamma_{b(Y)}} d g / g\right)=0$.

Proof. Since $1 / 2 \pi \int_{\Gamma_{j}} d g / g= \pm 1 / 2 \pi \int_{\Gamma_{j}^{\prime}} d g / g$, (3.4), it is $\pm i$ times the winding number of $g$ around $\Gamma_{\jmath}^{\prime}$, proving the first assertion. By (3.2), $\operatorname{Re}\left(1 / \pi \int_{\Gamma_{b(Y)}} d g / g\right)=\operatorname{Re}\left(1 / 2 \pi \int_{p^{-1}\left(\Gamma_{b}\right)} d g / g\right)$. Since $1 / 2 \pi i \int_{p^{-1}\left(\Gamma_{b}\right)} d g / g$ is the winding number of $g$ about $p^{-1}\left(\Gamma_{b}\right), \operatorname{Re}\left(1 / \pi \int_{\Gamma_{b\left(Y^{\prime}\right)}} d g / g\right)=0$, proving the proposition.

Using (3.8) we see that $\tilde{\lambda}$ (of (3.d)) induces a monomorphism $\check{\lambda}$ of $G \equiv\left(\mathscr{C}^{*}(\mathfrak{Y}) / \exp \mathscr{\mathscr { C }}(\mathfrak{Y})\right) /\{ \pm 1\}$ into $\Delta(\mathfrak{Y}) / d \mathscr{C}(\mathfrak{Y})$. Now recall that $\widetilde{\varphi}_{1}, \cdots, \widetilde{\varphi}_{2 b(Y)-1}$ are $R$-linear maps of $\Delta(\mathfrak{Y}) / d \mathscr{L}(\mathfrak{Y})$ into $R$. Let $\Phi \equiv$ $\left(\widetilde{\Phi}_{2}, \widetilde{\Phi}_{4}, \cdots, \widetilde{\Phi}_{2 b(Y)-2}\right)$ map $\Delta(\mathfrak{Y}) / d \mathscr{C}(\mathfrak{Y})$ into $R^{b(Y)-1}$ and let $\mu \equiv \Phi \circ \check{\lambda}$. By (3.9), $\mu$ maps $G$ into $Z^{b(Y)-1}$. 
THEOREM 3.10. $\mu$ is an isomorphism of $G\left(\equiv\left(\mathscr{X}^{*}(\mathfrak{Y}) / \exp \mathscr{K}(\mathfrak{Y})\right)\right) /$ $\{ \pm 1\})$ onto $Z^{b(Y)-1}$.

Proof. Let $f \in \mathscr{H}^{*}(\mathfrak{Y})$ and let $g$ be its residue in $G$. Assume that $\mu(g)=0$; then-by hypothesis $\varphi_{1}(\lambda(f)), \varphi_{2}(\lambda(f)), \cdots, \varphi_{2 b\left(Y^{\prime}\right)-1}(\lambda(f))=$ 0: i.e., $\widetilde{\varphi}_{1}(\tilde{\lambda}(f)), \widetilde{\varphi}_{2}(\widetilde{\lambda}(f)), \cdots, \widetilde{\varphi}_{2 b(Y)-1}(\widetilde{\lambda}(f))=0$. By $(3.6), \quad \tilde{\lambda}(f)=0$; thus $\check{\lambda}(g)=0$. Since $\check{\lambda}$ is injective, $g=0$, proving that $\mu$ is injective. By (2.6), $\{ \pm 1\}$ is the torsion group of $\mathscr{H}^{*}(\mathfrak{Y}) / \exp \mathscr{C}(\mathfrak{Y})$; thus $G$ is a free Abelian group of $\operatorname{rank} b(Y)-1,(2.5)$, which shows that $\mu$ is surjective.

We conclude by noting that $\mathscr{S}^{*}(\mathfrak{Y}) / \exp \mathscr{\mathscr { C }}(\mathfrak{Y})$ can be analyzed in the nonorientable case by noting that $\{ \pm 1\}$, its torsion group, reflects its nonorientability and that $\mu$ picks out the appropriate geometric "periods" about $\Gamma_{1}, \cdots, \Gamma_{b(Y)-1}$.

4. A mixed analytic and harmonic obstruction problem. As noted [4], the real part $\operatorname{Re} f$ of an analytic function $f$ on $\mathfrak{Y}$ is a welldefined harmonic function $u$ on $\mathfrak{Y}$. This may also be seen if we think of $f$ as an analytic function on $\mathfrak{x}$ fixed under $\sigma$, since if $f=u+i v$, $\sigma(f)=u \circ \tau-i v \circ \tau$; so $u$ is a real-valued harmonic function that is invariant under $\tau$ : i.e., $u$ engendered a real-valued harmonic function $u$ on $\mathfrak{Y}$. Let $L_{R}(\mathfrak{Y})$ denote the space of all real-valued harmonic functions on $\mathfrak{Y}$ and let $\operatorname{Re} \mathscr{H}(\mathfrak{Y}) \equiv\{\operatorname{Re} f: f \in \mathscr{C}(\mathfrak{Y})\}$. As noted above, $\operatorname{Re} \mathscr{H}(\mathfrak{Y})$ is a subspace of $\mathrm{L}_{R}(\mathfrak{Y})$. The question we treat in this section is the following: what is the dimension $m$, over $R$, of $L_{R}(\mathfrak{Y}) / \operatorname{Re} \mathscr{C}(\mathfrak{Y})$ ? In case $\mathfrak{Y}$ is orientable, $m$ is well-known to be $b(Y)$. (See, e.g., [1].) Thus assume, until further notice, that $Y$ is nonorientable.

As noted above, $L_{R}(\mathfrak{Y})$ can be considered as the subspace of $L_{R}(\mathfrak{X})$ whose elements are invariant under $\tau$. Given $u \in L_{R}(\mathfrak{Y})$, it has a harmonic differential du on $\mathfrak{X}$; thus we may form $\delta(u) \equiv \omega \equiv d u+i^{*} d u$, an analytic differential on $\mathfrak{X}$. Assume first that there exists $f \in \mathscr{X}$ (פ⿹) such that $f=u+i v$; then $d v=* d u$ and $\omega=d f$. Two facts emerge: (i) in this case $\omega \in \Delta(\mathfrak{Y})$, and (ii) $\omega$ is exact.

LEMmA 4.1. For all $u \in L_{R}(\mathfrak{V}), \delta(u) \equiv \omega$ is in $\Delta(\mathfrak{Y})$ : i.e., $\sigma(\omega)=\omega$.

Proof. Let $x^{\prime} \in X$ and let $\tau\left(x^{\prime}\right)=x^{\prime \prime}$. Since $\omega$ is an analytic differential on $\mathfrak{X}$ it is locally of the form $d g$ for some locally defined analytic function $g$ at $x^{\prime}$, where $g$ is of the form $u+i v$ locally at $x^{\prime}$. $\sigma(g)$ is an analytic function, defined locally at $x^{\prime \prime}$, of the form $u \circ \tau-i v \circ \tau$; thus the real part of $\sigma(\omega)$ at $x^{\prime \prime}$ is $d u$. Since this is true for all $x^{\prime} \in X$, the real part of $\sigma(\omega)$ is du globally. Since an analytic differential is uniquely determined by its real part, $\sigma(\omega)=\omega$, proving the lemma. 
The following sequence of $R$-spaces and $R$-linear maps is exact:

$$
0 \longrightarrow R \longrightarrow L_{R}(\mathfrak{Y}) \stackrel{\delta}{\longrightarrow} \Delta(\mathfrak{Y})
$$

It was also noted above that $\delta(\operatorname{Re} \mathscr{\mathscr { C }}(\mathfrak{Y})) \subset d \mathscr{Y}(\mathfrak{Y})$; thus $\delta$ induces an $R$-linear map $\tilde{\delta}$ of $L_{R}(\mathfrak{Y}) / \operatorname{Re} \mathscr{H}(\mathfrak{Y})$ into $\Delta(\mathfrak{Y}) / d \mathscr{C}(\mathfrak{Y})$.

LeMma 4.2. $\tilde{\delta}$ is injective.

Proof. Let $u \in L_{R}(\mathfrak{Y )})$ for which there exists $f \in \mathscr{H}$ (פ) such that $\omega=d f$. Let $f=a+b i, a$ and $b$ being real valued. Then $d u+i^{*} d u=$ $\omega=d a+i d b$; thus $d u=d a$, or $u=a+c$, $c$ being a real constant. Hence $u=\operatorname{Re}(f+c)$, proving that $u \in \operatorname{Re} \mathscr{Y}(\mathfrak{Y})$, proving the lemma.

Having found the kernel of $\tilde{\delta}$, namely zero, let us describe its cokernel. This will be done with the help of the linear functionals $\widetilde{\varphi}_{j}$ on $\Delta(\mathfrak{Y}) / d \mathscr{H}(\mathfrak{Y})$ developed in $\S 3$.

Lemma 4.3. For all $v \in L_{R}(\mathfrak{Y})$,

$$
\varphi_{1}(\delta(u))=\varphi_{3}(\delta(u))=\cdots=\varphi_{2 b(Y)-1}(\delta(u))=0 .
$$

Proof. The assertion is easily seen to boil down to the following statement: Given a piece-wise $C^{2}$-Jordan curve $\Gamma$ in $\mathfrak{X}, \int_{\Gamma} d u=0$, a fact which is obviously true, proving the lemma.

Using this rather crude lemma we obtain an upper bound on the cardinal number we are trying to compute.

COROLLARY 4.4. $\operatorname{dim}_{R} L_{R}(\mathfrak{Y}) / \operatorname{Re} \mathscr{H}(\mathfrak{Y}) \leqq b(Y)-1$.

LEMMA 4.5 Let $\omega \in \Delta(\mathfrak{Y})$ such that $\varphi_{1}(\omega), \varphi_{3}(\omega), \cdots, \varphi_{2 b\left(Y^{\prime}\right)-1}(\omega)=0$; then there exists $u \in L_{R}(\mathfrak{Y})$ such that $\delta(u)=\omega$.

Proof. The real part $\alpha$ of $\omega$ is a real harmonic differential on $\mathfrak{X}$ which is invariant under the map induced on such differentials by $\tau$. The conditions above assure that all of the periods of $\alpha$ are zero; thus it is of the form $d u$ for some $u \in L_{R}(\mathfrak{Y})$, proving the lemma.

In (3.6) we saw that $\left\{\widetilde{\varphi}_{1}, \widetilde{\varphi}_{2}, \cdots, \widetilde{\varphi}_{2 b(Y)-2}, \widetilde{\varphi}_{2 b(Y)-1}\right\}$ is a basis of $\operatorname{Hom}_{R}(\Delta(\mathfrak{Y}) / d \mathscr{H}(\mathfrak{Y}), R)$. Combining (4.3) and (4.5) with (3.6), we arrive at the main lemma of the section.

LeMmA 4.6. $\widetilde{\delta}\left(L_{R}(\mathfrak{Y}) / \operatorname{Re} \mathscr{\mathscr { C }}(\mathfrak{Y})\right)$ is the annihilator of the $R$-subspace of $\operatorname{Hom}_{R}(\Delta(\mathfrak{Y}) / d \mathscr{H}(\mathfrak{Y}), R)$ spanned by $\left\{\widetilde{\varphi}_{1}, \widetilde{\varphi}_{3}, \cdots, \widetilde{\varphi}_{2 b(Y)-3}, \widetilde{\varphi}_{2 b(Y)-1}\right\}$. 
Using this we have the main theorem of the section.

THeorem 4.7. $\operatorname{dim}_{R} L_{R}(\mathfrak{Y}) / \operatorname{Re} \mathscr{C}(\mathfrak{Y})=b(Y)-1$ if $Y$ is nonorientable, and is $b(Y)$ if $Y$ is orientable.

ExAmPLE 4.1. Let $\mathfrak{Y}$ be a noncompact Möbius strip; then every real-valued harmonic function on $\mathfrak{Y}$ is the real part of an $f$ in $\mathscr{\mathscr { C }}(\mathfrak{Y})$.

5. Application to the standard algebra on $\mathfrak{Y}$. Let $\mathfrak{Y}$ be a compact Klein surface whose boundary $\partial Y$ is nonempty. Let int $\mathfrak{Y}$ denote the interior of $\mathfrak{Y}$, with dianalytic structure. The standard algebra $A(\mathfrak{Y})$ on $\mathfrak{Y}$, defined and treated superficially in [2], is the algebra of all continuous complex "functions" on $\mathfrak{Y}$ that are analytic on int $\mathfrak{Y}$. Alternatively, we can define $A(\mathfrak{Y})$ using the orienting double $\mathfrak{X} \stackrel{p}{\longrightarrow} \mathfrak{Y}$ of $\mathfrak{Y}$, if $\mathfrak{Y}$ is nonorientable. (See [4] for details.) $\mathfrak{X}$ is then a compact orientable Klein surface and $p$ a two-to-one dianalytic covering map of $\mathfrak{X}$ onto $\mathfrak{Y}$, i.e., a morphism. Then $A(\mathfrak{Y})$ can be defined to be the set of all elements of the standard algebra on $\mathfrak{X}$ invariant under $\sigma$, where $\sigma(f)=\kappa \circ f \circ \tau$. Let $f \in A(\mathfrak{Y})$ be represented as $u+i v$, $u$ and $v$ being real-valued harmonic functions on $\mathfrak{X}$. $u$ is then continuous on $Y$ and harmonic on int $\mathfrak{Y}$. Let $\operatorname{Ref} \equiv u \mid \partial Y$, and note that Re is an $R$-linear map of $A(\mathfrak{Y})$ into $C_{R}(\partial Y)$, the $R$-algebra of all continuous real-valued functions on $\partial Y$. Under the sup norm, $C_{R}(\partial Y)$ is a real Banach algebra. Let $\operatorname{cl} \operatorname{Re} A(\mathfrak{Y})$ denote the closure of $\operatorname{Re} A(\mathfrak{Y})$ in $C_{R}(\partial Y)$ under the sup norm. Let D.d. $(A(\mathfrak{Y})) \equiv$ $\operatorname{dim}_{R} C_{R}(\partial Y) / \mathrm{cl} \operatorname{Re} A(\mathfrak{Y})$ be known as the Dirichlet deficiency of $A(\mathfrak{Y})$. $\quad A(\mathfrak{Y})$ will be called a hypodirichlet algebra if $\mathrm{D} . \mathrm{d} .(A(\mathfrak{Y}))$ is finite, and a Dirichlet algebra if D. d. $(A(\mathfrak{Y}))=0$. This notion is well-known for function algebras.

In the orientable case, Wermer [12] has shown that $A(\mathfrak{Y})$ is a hypodirichlet algebra whose Dirichlet deficiency is bounded above by $b(Y)$, the first Betti number of $Y$, with a strong suggestion that equality occurs. (For a proof that D. d. $(A(\mathfrak{Y}))=b(Y)$ see, e.g., [1].) The object of this section is to show, in the nonorientable case, that $A(\mathfrak{Y})$ is a hypodirichlet algebra, and to compute $\mathrm{D}$. d。 $A(\mathfrak{Y}))$ ). In so doing we follow very much in Wermer's footsteps, except for a detour or two occasioned by the nonorientable terrain.

Let $g \in C_{R}(\partial Y)$, and regard $g$ as a symmetric element $g$ of $C_{R}(\partial X)$ : i.e., $g \circ \tau=g$. Using the Dirichlet principle, valid on $\mathfrak{X}$, there exists a continuous real-valued function $W$ on $X$, harmonic on int $\mathfrak{X}$ such that $W \mid \partial Y=g$. Let $U \equiv(W+W \circ \tau) / 2$. Note that $U$ is continuous on $X$, harmonic on int $\mathfrak{X}, U \mid \partial X=g$, and $U \circ \tau=U$. (Of course by uniqueness $U=W$.) Thus we have proved the following. 
Lemma 5.1. The Dirichlet principle is valid on $\mathfrak{Y}$.

Let har $g \equiv U \mid$ int $Y \equiv u$; then har is an $R$-monomorphism of $C_{R}(\partial Y)$ into $L_{R}($ int $\mathfrak{Y})$, the space of all real-valued harmonic functions on int $\mathfrak{Y}$.

Recall that $\delta$, defined in $\S 4$, takes $u \in L_{R}$ (int $\left.\mathfrak{Y}\right)$ to $d u+i^{*} d u \equiv$ $\omega \in \Omega($ int $\mathfrak{X})$ which turns out $(4.1)$ to be in $\Delta$ (int $\mathfrak{Y})$. Thus $\eta_{j}: g \rightarrow \varphi_{j}$ $(\delta($ har $(g)))$ are linear functionals on $C_{R}(\partial Y), 1 \leqq j \leqq 2 b(Y)-1$. By (4.3), $\eta_{1}, \eta_{3}, \cdots, \eta_{2 b(Y)-1}$ are zero.

LemmA 5.2. If $g \in \operatorname{Re} A(\mathfrak{Y})$, then $\eta_{2},(g), \eta_{4}(g), \cdots, \eta_{2 b(Y)-2}(g)=0$.

Proof. Let $f \in A(\mathfrak{Y}) \subset A(\mathfrak{X})$ be written in the form $u+i v$ on $X, u$ and $v$ real-valued functions, $u \mid \operatorname{int} \mathfrak{X}=\operatorname{har} \operatorname{Re} f$, and $\delta($ har $\operatorname{Re} f)=$ $d u+i * d u$, on int $\mathfrak{X}$, equals $d u+i d v=d f$ on int $\mathfrak{X}$. Since all the periods of $d f$ are zero, we may use (3.5) to conclude that $\eta_{2}(g)=$ $\eta_{4}(g)=\cdots=\eta_{2 b(Y)-2}(g)=0$.

From (5.2) we conclude that $\operatorname{Re} A(\mathfrak{Y})$ is contained in $H$, the hyperplane of $C_{R}(\partial Y)$ of functions annihilated by $\eta_{2}, \eta_{4}, \cdots, \eta_{2 b(Y)-2}$. In order to be able to conclude that $\operatorname{cl} \operatorname{Re} A(\mathfrak{Y}) \subset H$, it suffices to prove the following.

Lemma 5.3. $\eta_{2}, \eta_{4}, \cdots, \eta_{2 b(Y)-2}$ are continuous on $C_{R}(\partial Y)$ (under the sup norm topology).

Proof. Let $\left(g_{n}\right)_{n \in N}$ be a null sequence in $C_{R}(\partial Y)$ and let $U_{n}$ be the real-valued continuous extension of $g_{n}$ to $X$ which is harmonic on int $\mathfrak{X}$. Using the maximum principle $\left(U_{n}\right)_{n \in N}$ is uniformly a null sequence on $X$. Let 3 be a tubular neighborhood of $\Gamma_{j}^{\prime}, 1 \leqq j \leqq$ $b(Y)-1$, which is an annular compact bordered Riemann surface. Let 3 be embedded in $C$ as an annulus $\mathfrak{W}_{r} \equiv\{z \in C: r \leqq|z| \leqq 1 / r\}$ for some $r, 0<r<1$. Thus, without loss of generality, we may assume that $\mathfrak{X}=\mathfrak{W}_{r}$. $\quad U_{n}$ is obtained by integrating $g_{n}$ times the normal derivative of the appropriate Green's functions on $\mathfrak{X} . \partial U_{n} / \partial x$ and $\partial U_{n} / \partial y$ are obtained by integrating $g_{n}$ times the appropriate derivative of this kernel; thus $\partial U_{n} / \partial x$ and $\partial U_{n} / \partial y$ tend uniformly to zero. Thus $1 / 2 \pi i \int_{\Gamma_{j}^{\prime}} * d U_{n}$ tends to zero, proving the lemma.

COROLlaRY 5.4. $H$ is a closed hyperplane of $C_{R}(\partial Y)$; thus cl $\operatorname{Re} A(\mathfrak{Y}) \subset H$ and D.d. $A(\mathfrak{Y}) \geqq b(Y)-1$.

Lemma 5.5. Let $g$ be a $C^{2}$-function in $H$. There exists $f \in A$ (श)) 
such that $\operatorname{Re} f=g$; hence $H=\operatorname{cl} \operatorname{Re} A(\mathfrak{Y})$.

Proof. Let $\omega \equiv \delta(g)$; then $\omega \in \Delta($ int $\mathfrak{Y})$. Since $g \in H$, $\omega$ is without periods on $\mathfrak{X}$. By (3.5), there exists $f$ analytic on int $\mathfrak{Y}$ such that $\omega=d f$. Let $a$ be the real part of $f$; then $d a=d u$, where $u=$ har $g$. $a$ and $u$ differ by a real constant, which we may assume is zero. Since $g$ is $C^{2}$ on $\partial Y, f$ extends to a unique element $f \in A(\mathfrak{Y})$. Clearly $\operatorname{Re} f=g$. Since the $C^{2}$-functions in $C_{R}(\partial Y)$ are dense, $H=\operatorname{cl} \operatorname{Re} A(\mathfrak{Y})$, proving the lemma.

We come then to the main theorem of the section.

THEOREM 5.6. Let $\mathfrak{Y}$ be a compact Klein surface. A(Y) is a hypodirichlet algebra whose Dirichlet deficiency is $b(Y)$ if $\mathfrak{Y}$ is orientable and $b(Y)-1$ if $\mathfrak{Y}$ is nonorientable.

Wermer [12] goes on to prove somewhat more in the orientable case; namely that there exist $f_{1}, \cdots, f_{b(Y)} \in A^{*}(\mathfrak{Y})$, the group of units of $A(\mathfrak{Y})$, such that the real vector space spanned by $\operatorname{Re} A(\mathfrak{Y}) \cup\left\{\log \left|f_{1}\right|\right.$, $\left.\cdots, \log \left|f_{b(Y)}\right|\right\}$ is dense in $C_{R}(\partial Y)$. Let us consider this in case $\mathfrak{Y}$ is nonorientable. First note that even though $f \in A^{*}(\mathfrak{Y})$ may not be a function on $Y,|f|$ is a function on $Y$ : i.e., considered as a function on $X,|f|$ is invariant under $\tau$.

Theorem 5.7. Assume that $\mathfrak{Y}$ is nonorientable. There exist $f_{1}, \cdots, f_{b(Y)-1} \in A^{*}(\mathfrak{Y})$ such that the real vector space spanned by $\operatorname{Re} A(\mathfrak{Y}) \cup\left\{\log \left|f_{1}\right|, \cdots, \log \left|f_{b\left(Y^{Y}\right)-1}\right|\right\}$ in $C_{R}(\partial Y)$ is dense in $C_{R}(\partial Y)$. Further the images of $\log \left|f_{1}\right|, \ldots, \log \left|f_{b\left(Y^{\prime}\right)-1}\right|$ in $V \equiv C_{R}(\partial Y) / H$ form a basis of $V$ over $R$.

Proof. Proceeding much as Wermer did [12], let $\mathfrak{X}$ be analytically embedded in a slightly larger noncompact Riemann surface $\mathfrak{B}$ in such a way that $\Gamma_{1}^{\prime}, \Gamma_{1}^{\prime \prime}, \Gamma_{2}^{\prime}, \Gamma_{2}^{\prime \prime}, \ldots, \Gamma_{b(Y)-1}^{\prime}, \Gamma_{b(Y)-1}^{\prime \prime}$, and $p^{-1}\left(\Gamma_{b(Y)}\right)$ are still a basis for the first singular homology group on $W$. By (3.6) and (4.6), there exist $u_{1}, \cdots, u_{b(Y)-1} \in L_{R}(\mathfrak{B})$, invariant under $\tau$, such that $\varphi_{2 j}\left(\delta\left(v_{k}\right)\right)=\delta_{j k}, 1 \leqq j, k \leqq b(Y)-1$. Let $\omega_{k} \equiv \delta\left(v_{k}\right), 1 \leqq k \leqq b(Y)-1$. Let $x_{0} \in p^{-1}\left(I_{b(Y)}\right)$ and consider expressions $\exp \left(2 \pi \int_{x_{0}}^{x} \omega_{k}-\pi \int_{x_{0}}^{\tau\left(x_{0}\right)} \omega_{k}\right)$, where the last integral is taken via a fixed Jordan arc $\Gamma$ on $X$ from $x_{0}$ to $\tau\left(x_{0}\right)$. Recall that $\omega_{k} \equiv d u_{k}+i * d u_{k}$; thus $\omega_{k}$ has no real periods, only imaginary periods. By the choice of $u_{k},{ }^{*} d u_{k}$ has only integral periods; thus the expression above is a well defined analytic function $f_{k}(x)$ on W. We now wish to show that $\sigma\left(f_{k}\right)=f_{k}$. 


$$
\begin{aligned}
\sigma\left(f_{k}\right)(x) & =\kappa \circ \exp \left(2 \pi \int_{x_{0}}^{\tau(x)} \omega_{k}-\pi \int_{x_{0}}^{\tau\left(x_{0}\right)} \omega_{k}\right) \\
& =\kappa \circ \exp \left(2 \pi \int_{x_{0}}^{\tau\left(x_{0}\right)} \omega_{k}+2 \pi \int_{\tau\left(x_{\hat{\vartheta}}\right)}^{\tau(x)} \omega_{k}-\pi \int_{x_{0}}^{\tau\left(x_{0}\right)} \omega_{k}\right) \\
& =\kappa \circ \exp \left(2 \pi \int_{\tau\left(x_{0}\right)}^{\tau(x)} \omega_{k}+\pi \int_{x_{0}}^{\tau\left(x_{0}\right)} \omega_{k}\right) \\
& =\exp \left(2 \pi \int_{x_{0}}^{x} \omega_{k}+\pi \kappa \circ \int_{x_{0}}^{\tau\left(x_{0}\right)} \omega_{k}\right) .
\end{aligned}
$$

Since $x_{0} \in p^{-1}\left(\Gamma_{b(Y)}\right), \tau\left(x_{0}\right)$ is necessarily in this set; thus we may assume that $\Gamma$ is a subarc of $p^{-1}\left(\Gamma_{b(Y)}\right)$ and hence that $\Gamma+\tau(\Gamma)=$ $p^{-1}\left(\Gamma_{b(Y)}\right)$. By (3.2) $\int_{p^{-1}\left(\Gamma_{b(Y)}\right)} \omega_{k} \equiv a$ is real. By definition (3.b) and by (3.4), $a=2 \pi \Phi_{2 b(Y)-1}\left(\omega_{k}\right)$. Since $\omega_{k}=\delta\left(u_{k}\right)$, and $u_{k} \in L_{R}(\mathfrak{Y})$, we may apply (4.3) and conclude that $a=0 . \operatorname{By}(3.1) \int_{\Gamma} \omega_{k}=\kappa \circ \int_{\tau(\Gamma)} \omega_{k}$; thus

$$
0=\int_{p^{-1}\left(\Gamma_{b(Y)}\right)} \omega_{k}=\int_{\Gamma} \omega_{k}+\int_{\tau(\Gamma)} \omega_{k}=\int_{\Gamma} \omega_{k}+\kappa \circ \int_{\Gamma}\left(\omega_{k}\right)=2 \operatorname{Re} \int_{\Gamma} \omega_{k} .
$$

Hence $\int_{\Gamma} \omega_{k}\left(\equiv \int_{x_{0}}^{\tau\left(x_{0}\right)} \omega_{k}\right)$ is purely imaginary. As a consequence $\kappa \circ \int_{x_{0}}^{\tau\left(x_{0}\right)} \omega_{k}=$ $-\int_{x_{0}}^{\tau\left(x_{0}\right)} \omega_{k}$ and $\sigma\left(f_{k}\right)(x)=f_{k}(x)$ for all $x \in W$. This shows that $f_{k}$ is invariant under $\sigma$ and thus when restricted to $\mathfrak{X}$ is in $A^{*}(\mathfrak{Y})$. $\log \left|f_{k}(x)\right|=2 \pi \int_{x_{0}}^{x} d u_{k}=2 \pi\left(u_{k}(x)-u_{k}\left(x_{0}\right)\right)$; thus $\varphi_{2 j}\left(\delta\left(\log \left|f_{k}\right|\right)\right)=2 \pi \delta_{j k}$ for $1 \leqq j, k \leqq b(Y)-1$. The rest follows from (4.2), (4.6), (3.6), and $(5.5)$.

Note. The author is indebted to Newcomb Greenleaf for suggesting, in a different context (namely in [4, I, $\S 10]$ ), the trick of subtracting $\int_{x_{0}}^{\tau\left(x_{0}\right)} \omega_{k}$.

In case $\mathfrak{Y}$ Is orientable it is essentially well-known, and was noted in $[1, \S 9]$, that the real linear span of $\log \left|A^{*}(\mathfrak{Y})\right|$ is dense in $C_{R}(\partial Y)$ : i.e., $A^{*}(\mathfrak{Y})$ is an Arens-Singer algebra [1, §9]. Thus we have the following.

COROLlary 5.8. A(Y) is an Arens-Singer algebra whether $\mathfrak{Y}$ is orientable or not: i.e., the real linear span of $\log \left|A^{*}(\mathfrak{Y})\right|$ in $C_{R}(\partial Y)$ is dense in $C_{R}(\partial Y)$.

6. Conclusion. As noted in the synopsis, the purpose of this paper was not only to study the analytic and harmonic obstruction on nonorientable Klein surfaces $\mathfrak{Y}$, which resembles the orientable 
case very closely, but to try to elucidate the reason why the defect of minus 1, which we will call the Klein defect, occurs. The de Rham problem $(0 . b)$ on $\mathfrak{Y}$ occasioned our first insight into the reason that the Klein defect occurs, when we note that when $Y$ is doubled to form $X$ each untwisted strip doubles, whereas if $m=1$ this untwisted strip gives rise to only one strip on $X$, and if $m=2$ these two twisted strips give rise to three strips on $X$ (2.c). The reason that the Klein defect occurs in the units modulo exponentials problem (0.e) lies a bit deeper, but may be seen through the language of Čech cohomology in Examples 2.1 and 2.2, and (2.4).

Integration theory was introduced in $\S 3$ to see how the Klein defect manifests itself in this more classical setting and to develop a powerful method thereby. The prime fact is that $\operatorname{Im} \int_{p^{-1}\left(\Gamma_{b(Y)}\right)} \omega=0$ for all $\omega \in \Delta(\mathfrak{Y )})$, (3.2). In [4] we saw that only the real part of $\int_{\Gamma_{b(Y)}} \omega$ is well defined, the imaginary part being without invariancenot even being defined up to sign. This "missing" imaginary period results in the emergence of the Klein defect in (3.6), (3.10), (4.6), (4.7), (5.6), and (5.7): i.e., in the rest of the main theorems of the paper. The presence of two torsion in $\mathscr{K}^{*}(\mathfrak{Y}) / \exp \mathscr{\mathscr { C }}(\mathfrak{Y})$ is not detected by integration theory in this paper, since $\lambda(g) \equiv d g / g=\lambda(-g)$. The winding numbers of $g$ around the untwisted strips is, however, recaptured by integration in (3.10). The two torsion in this context is thus seen as only arithmetic, even though in the language of Čech cohomology it does manifest itself geometrically.

Not only has an instance of torsion occurring in analysis been found, but the answers to each of these analysis problems; the de Rham problem, (2.5) and the units modulo exponentials problem (2.5); distinguish between the orientable and the nonorientable case; thus each of these problems (and also $H^{1}(Y, \mathscr{K})$ and $H^{1}(Y, \mathscr{G})$ ) are more sensitive and revealing of the topology on $\mathfrak{Y}$ than anything in homotopy theory, or than any homology or cohomology theory for which the homotopy axiom [5] holds. Similarly, given the rank $b(Y)$ say of the first homology group of $Y$ and the solution of either the harmonic functions modulo the real part of an analytic function problem (4.7), or the Dirichlet deficiency problem (5.6), more is revealed about the topology on $Y$ than by all of algebraic topology which is homotopy invariant.

Thanks are due to Newcomb Greenleaf with whom the author consulted on several occasions, and whose criticisms of the penultimate version the manuscript resulted in a number of important changes. Thanks are also due to Burt Rodin with whom the author conversed 
oft and many a time while in La Jolla. Particular thanks are due to Helmut Röhrl who helped make the author's very productive stay in La Jolla, California possible; this paper having been largely written there within sight of the Pacific.

\section{REFERENCES}

1. N. L. Alling, Extensions of meromorphic function rings over noncompact Riemann surfaces, II, Math. Z. 93 (1966), 345-394.

2. - Real Banach algebras and nonorientable Klein surfaces, Crella 241 (1970), 200-208.

3. N. L. Alling and N. Greenleaf, Klein surfaces and real algebraic function fields, Bull. Amer. Math. Soc. 75 (1969), 869-872.

4. - Foundations of the theory of Klein surfaces and real algebraic function fields, Springer-Verlag's Lecture Notes in Mathematics (to appear)

5. S. Eilenberg and N. Steenrod, Foundations of Algebraic Topology, Princeton University Press, Princeton, N J., 1952.

6. H. Florack, Reguläre und meromorphe Funktionen auf nicht geschlossenen Riemannschen Flächen, Math. Inst. Univ. Münster 1 (1948).

7. N. Greenleaf, Analytic sheaves on Klein surfaces (submitted for publication)

8. R. C. Gunning and H. Rossi, Analytic functions of several complex variables, Prentice-Hall, Englewood Cliffs, N. J., 1965.

9. W. S. Massey, Algebraic Topology: An Introduction, Harcourt, Brace and World, 1967.

10. R. Redheffer, Homotopy theorems of function theory, Amer. Math. Monthly 76 (1969) 778-787.

11. M. Schiffer and D. Spencer, Functionals of Finite Riemann surfaces, Princeton University Press, Princeton, N. J., 1954.

12. J. Wermer, On algebras of continuous functions, Proc. Amer. Math. Soc. 4 (1953), 866-869.

Received May 5, 1970. This research was supported, in part, by NSF Grant GP 9214 while the author was on subbatical leave from the University of Rochester at the University of California, San Diego.

UNIVERSITY OF ROCHESTER

Rochester, New York 



\title{
PACIFIC JOURNAL OF MATHEMATICS
}

\author{
EDITORS
}

H. SAMELSON

Stanford University

Stanford, California 94305

C. R. Новву

University of Washington

Seattle, Washington 98105
J. DUGUNDJI

Department of Mathematics

University of Southern California

Los Angeles, California 90007

RICHARD ARENS

University of California

Los Angeles, California 90024

\section{ASSOCIATE EDITORS}

E. F. BECKENBACH

B. H. NeUManN

F. WOLF

K. YosHidA

\section{SUPPORTING INSTITUTIONS}

UNIVERSITY OF BRITISH COLUMBIA CALIFORNIA INSTITUTE OF TECHNOLOGY UNIVERSITY OF CALIFORNIA MONTANA STATE UNIVERSITY UNIVERSITY OF NEVADA NEW MEXICO STATE UNIVERSITY OREGON STATE UNIVERSITY UNIVERSITY OF OREGON OSAKA UNIVERSITY UNIVERSITY OF SOUTHERN CALIFORNIA
STANFORD UNIVERSITY UNIVERSITY OF TOKYO UNIVERSITY OF UTAH WASHINGTON STATE UNIVERSITY UNIVERSITY OF WASHINGTON

AMERICAN MATHEMATICAL SOCIETY CHEVRON RESEARCH CORPORATION TRW SYSTEMS

NAVAL WEAPONS CENTER 


\section{Pacific Journal of Mathematics}

\section{Vol. 36, No. $1 \quad$ November, 1971}

Norman Larrabee Alling, Analytic and harmonic obstruction on

nonorientable Klein surfaces ............................ 1

Shimshon A. Amitsur, Embeddings in matrix rings .............. 21

William Louis Armacost, The Frobenius reciprocity theorem and essentially bounded induced representations ....................... 31

Kenneth Paul Baclawski and Kenneth Kapp, Topisms and induced

non-associative systems ............................ 45

George M. Bergman, The index of a group in a semigroup ............ 55

Simeon M. Berman, Excursions above high levels for stationary Gaussian

processes....................................... 63

Peter Southcott Bullen, A criterion for $n$-convexity .............. 81

W. Homer Carlisle, III, Residual finiteness of finitely generated commutative

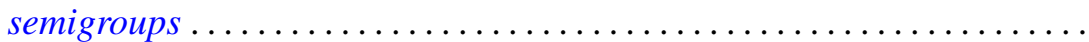

Roger Clement Crocker, On the sum of a prime and of two powers of

two ............................................ 103

David Eisenbud and Phillip Alan Griffith, The structure of serial rings . . . 109

Timothy V. Fossum, Characters and orthogonality in Frobenius

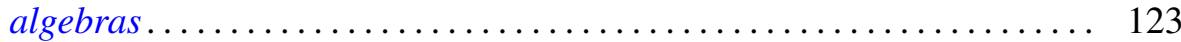

Hugh Gordon, Rings of functions determined by zero-sets . .......... 133

William Ray Hare, Jr. and John Willis Kenelly, Characterizations of Radon partitions...

Philip Hartman, On third order, nonlinear, singular boundary value

problems

David Michael Henry, Conditions for countable bases in spaces of

countable and point-countable type ..

James R. Holub, Hilbertian operators and reflexive tensor products ...

Robert P. Kaufman, Lacunary series and probability ..... . .

195

Erwin Kreyszig, On Bergman operators for partial differential equations in

two variables ................................

Chin-pi Lu, Local rings with noetherian filtrations . .

Louis Edward Narens, A nonstandard proof of the Jordan curve theorem...

S. P. Philipp, Victor Lenard Shapiro and William Hall Sills, The Abel summability of conjugate multiple Fourier-Stieltjes integrals. .

Joseph Earl Valentine and Stanley G. Wayment, Wilson angles in linear normed spaces

Hoyt D. Warner, Finite primes in simple algebras ...

Horst Günter Zimmer, An elementary proof of the Riemann hypothesis for an elliptic curve over a finite field... 